American Journal of Immunology 5 (4): 101-107, 2009

ISSN 1553-619X

(C) 2009 Science Publications

\title{
Foxp3-Mediated Immunity of Human Pancreatic Cancer Cell Line PANC-1
}

\author{
${ }^{1,3}$ Renxi Wang, ${ }^{1,3}$ Gencheng Han, ${ }^{1}$ Jianan Wang, ${ }^{1}$ Guojiang Chen, ${ }^{1}$ Ruonan Xu, \\ ${ }^{1}$ Liyan Wang, ${ }^{2} \mathrm{Xia} \mathrm{Li},{ }^{1}$ Beifen Shen and ${ }^{1}$ Yan Li \\ ${ }^{1}$ Department of Molecular Immunology, Institute of Basic Medical Sciences, \\ Taiping Road, No. 27, Beijing 100850, People's Republic of China \\ ${ }^{2}$ Institute of Immunology, Medical School of Henan University, \\ Kaifeng, People's Republic of China
}

\begin{abstract}
Problem statement: More and more evidences have shown that human cancer cells can express Foxp3, the regulatory T cell-associated transcription factor. The role of Foxp3 in human cancer cells is still unclear. We detected Foxp3 expression in human pancreatic cancer cell line PANC-1. SiRNA assays showed that Foxp3 expression suppressed the expression of TGF- $\beta$ and IL-10 which could induce immune tolerance. Approach: Thus, we proposed that Foxp3 expression in PANC-1 cells controlled the response but not tolerance characteristics of the cells. To prove the proposal, we first developed the PANC-1 cell-induced the immune response model. PANC-1 cells induced response in autoimmune Non-Obese Diabetes (NOD) mice aged of $>12$ weeks. In vitro cell co-cultured assay demonstrated that PANC-1 induced response in splenocytes from NOD mice aged of $>12$ weeks. Results: In the same time, we found that inflammatory cytokine such as INF- $\gamma$, IL-2 and IL-17 increased and anti-inflammatory cytokine such as IL-4, IL-10 and TGF- $\beta$ decreased in the co-cultured supernatant. These results demonstrate that PANC-1 cells induced response in splenocytes from NOD mice aged of $>12$ weeks. SiRNA assays showed that Foxp3 in PANC-1 cells controlled the response in the co-cultured cells. Conclusion: The results suggested that Foxp3 control the immunological response characteristics of PANC-1 cells in autoimmune condition.
\end{abstract}

Key words: PANC-1, tolerance, response, nod mice, foxp3

\section{INTRODUCTION}

Foxp3 has been regarded as a key factor in regulatory $\mathrm{T}$ cell (Treg) $^{[1]}$. Mutations in Foxp3 completely omit Treg in mouse and greatly reduce suppressive activity of Treg in human ${ }^{[2]}$. Moreover, ectopic expression of Foxp3 makes conventional murine $\mathrm{T}$ cells to gain the function of $\mathrm{Treg}^{[3]}$, and human $\mathrm{T}$ cells to acquire many characteristics of Treg $^{[4,5]}$. The molecular mechanisms through which Foxp3 determined the function and development of Treg are now studied by many groups. Foxp3 can form a complex with the nuclear factor of activated $\mathrm{T}$ (NFAT) cells and regulates the transcription of several target genes including interleukin $2^{[6]}$. The results are consent with the characteristics of Foxp3 as suppressive transcription factor.

Until recently, Foxp3 expression is found only in suppressive $\mathrm{T}$ cells including $\mathrm{CD} 4^{+} \mathrm{Foxp} 3^{+}$Treg, and CD $8^{+}$Foxp $3^{+}$Treg. Now, there are more and more evidences suggesting that human tumor cells also expressed Foxp $3^{[7-9]}$. The function of Foxp3 in human tumor cells is studied by many groups. In breast cancer, Foxp3 was regarded as an important suppressor for human breast cancer by suppressing HER-2/ErbB2 oncogene expression ${ }^{[10]}$. Another paper found that Foxp3-expressing pancreatic carcinoma cell line PANC-89 (also T3M4) suppressed T-cell proliferation, and knochdown of Foxp3 using RNA interference could effectively reduce the suppressive activity of these cells by up-regulation of IL- 6 and IL- $8^{[7]}$.

The role of Foxp3 in human tumor cells may be different in different tumor cells. This may be in fact that Foxp3 as suppressive transcription factor suppresses the different targeted molecular in different tumor cells. Previous study has found that PANC-89 expresses IL-6 but not TGF- $\beta$, whereas PANC-1 expresses TGF- $\beta$ but not IL- $6^{[11]}$. Thus, we propose that if it was expressed in PANC-1 cells, Foxp3 may be an important factor inducing tumor immunity by suppressing TGF- $\beta$ expression. We here study the proposal.

Corresponding Author: Yan Li, Department of Molecular Immunology, Institute of Basic Medical Sciences, Taiping Road, No.27, Beijing, 100850, People's Republic of China 


\section{MATERIALS AND METHODS}

Mice: NOD were obtained from the Jackson Laboratory and bred in our animal facilities under specific pathogen-free conditions. In female NOD mice, spontaneous diabetes began to appear by 12 -weeks of age (increasing to $80-90 \%$ incidence at 30 weeks of age). Mice were screened for glucose levels every week and considered diabetic when glucose levels were $\geq 13.8 \mathrm{mMol} \mathrm{L}^{-1}$. This study was approved by the Animal Care and Use Committee of the Beijing Institute of Basic Medical Science.

Antigen preparation: We predicted T-cell epitope of Glutamic Acid Decarboxylase 65 (GAD 65) and proved that $\mathrm{GAD}_{500-585}$ (representing the corresponding amino acids of GAD65) was a main T-cell epitope ${ }^{[24,25]}$. Antigen preparation has been described previously ${ }^{[24]}$. Briefly, the recombinant peptide $\mathrm{GAD}_{500-585}$ was produced from Escherichia coli BL-21 containing the bacterial expression vector pET28a $^{+}$vector. Recombinant $\mathrm{GAD}_{500-585}$ was purified using $\mathrm{Co}^{2+} /$ TALONIMAC resin (Clontech) according to the manufacturer's instructions. Eluted $\mathrm{GAD}_{500-585}$ fractions were dialyzed against PBS (pH7.2).

Cell preparation: Human pancreatic carcinoma cell lines PANC-1 (American Type Culture Collection, Manassas, VA) were grown in RPMI (Biochrom, Berlin, Germany) 10\% FCS (Atlanta Biologicals, Atlanta, GA). Lymphocytes from the spleen were collected under sterile conditions, obtained with mouse Ficoll separation liquid, washed twice with an incomplete RPMI medium and then re-suspended at $5 \times 10^{6}$ cells $\mathrm{mL}^{-1}$ in $10 \%$ FCS in an RPMI 1640 medium.

In vitro proliferation: For analysis of cellular response, lymphocytes were collected and cultured $\left(5 \times 10^{5}\right.$ cells/well) in triplicate in $0.1 \mathrm{ml}$ of RPMI-1640 medium supplement with $1 \%$ horse serum and restimulated with $30 \mu \mathrm{g} \mathrm{mL}^{-1} \mathrm{GAD}_{500-585}$ without or in the presence of PANC-1 or Foxp $3^{-1}$ PANC- 1 cells $\left(5 \times 10^{4}\right.$ cells/well). On day 3 , cultures were pulsed with $1 \mu \mathrm{Ci} /$ well of $\left[{ }^{3} \mathrm{H}\right]$ thymidine for the last $16 \mathrm{~h}$, and the cells harvested and counted by standard liquid scintillation. The results were expressed as the stimulation index (SI, c.p.m. with antigen divided by c.p.m. with medium alone) or CPM.

Determination of cytokines production: The splenocytes were collected and cultured as for lymphocyte proliferation assay. Supernatants of the cells were harvested $24 \mathrm{~h}$ later for IL-2 and $48 \mathrm{~h}$ later for IFN- $\gamma$, IL-17, IL-4, IL-10 and TGF- $\beta 1$ assay using a sandwich ELISA. The ELISA kits used in this study were purchased from Biosource (IL-2), R and D (TGF$\beta 1$ ), eBioscience (IL-17A), and BD Pharmingen (IFN- $\gamma$, IL-4, IL-10), respectively.

Flow cytometry: Cells $\left(1 \times 10^{5}\right.$ cells/sample) were washed with fluorescence-activated cell sorting staining buffer (phosphate-buffered saline, 2\% fetal bovine serum or 1\% bovine serum albumin, $0.1 \%$ sodium azide). All samples were incubated with the $2.4 \mathrm{G} 2$ anti-Fc receptors (BD Pharmingen), prior to incubation with other Abs diluted in fluorescence-activated cell sorting buffer supplemented with $2 \%$ anti-Fc receptor $\mathrm{Ab}$. For the intracellular staining of Foxp3 protein, fixed cells were permeabilized with Perm Buffer (eBioscience) for $1 \mathrm{~h}$ at $4^{\circ} \mathrm{C}$. Cells were incubated for $30 \mathrm{~min}$ at $4^{\circ} \mathrm{C}$ with phycoerythrinconjugated anti-human Foxp3 $\mathrm{Ab}$ or isotype $\mathrm{Ab}$ (eBioscience, San Diego, CA, USA) at the concentration recommended by the manufacturer, washed, and analyzed by flow cytometry. Cells were washed and fixed with $1 \%$ paraformaldehyde prior to analysis in a FACSCalibur flow cytometer using CellQuest version 3.3 software (BD Biosciences, San Jose, CA).

Introduction of Foxp3-siRNA into PANC-1 cells: To introduce the human Foxp3 siRNAs (SC-43569, Santa Cruz Biotechnology, INC) into cultured PANC-1 cells, we used Lipofectamine Reagent and Plus Reagent (Invitrogen, Carlsbad, CA, USA) according to the manufacturer's procedure. Briefly, siRNAs were incubated with the Plus Reagent in serum-free DMEM for $15 \mathrm{~min}$ at room temperature, and then $350 \mu \mathrm{l}$ of the mixture was incubated with an equal volume of the Lipofectamine reagent $(16.8 \mu \mathrm{g})$ for another $15 \mathrm{~min}$ in order for them to form the complex of the siRNA with liposome. The PANC-1 cells on a $35 \times 10 \mathrm{~mm}$ Petri dish (Becton Dickinson Labware, Franklin Lakes, NJ, USA) were then incubated in the mixture of siRNAs with the Lipofectamine reagents for $6 \mathrm{~h}$ at $37^{\circ} \mathrm{C}$, then added to $700 \mu \mathrm{L}$ of DMEM containing 10\% FBS and incubated for another $42 \mathrm{~h}$. To confirm the introduction of Foxp3siRNA into cultured PANC-1 cells, cells were stained with PE-conjugated anti-human Foxp3 and analyzed by flow cytometry.

Statistical analysis: Results of cytokine ELISA were analyzed by the Student's t-test. Differences in diabetes incidence were analyzed by $\chi$ quadrate test. The cumulative diabetes onset was compared by KaplanMeier analysis. All differences reported in the results were significant $(\mathrm{p}<0.05)$. 


\section{RESULTS}

Foxp3 expression in PANC-1 cells: Despite many advances in understanding the mechanisms leading to tumor immunity, malignant tumors are tolerated, progress, and ultimately kill their host. Tumor cells employed different strategies to thwart immune responses: tumor-induced impairment of antigen presentation, the activation of negative co-stimulatory signals, and the elaboration of immunosuppressive factors $^{[12]}$. IL-10 and TGF- $\beta$ synthesized by tumor which exerts suppressive effects on the immune system $^{[12-14]}$. We found PANC-1 cells also secreted high level of TGF- $\beta$ and low level of IL-10 (Fig. 1A). In vitro studies have shown that TGF- $\beta$ can impose a regulatory phenotype on $\mathrm{CD} 4^{+} \mathrm{CD} 25^{-} \mathrm{T}$ cells through the induction of Foxp3 expression ${ }^{[15,16]}$. This makes us propose that PANC-1 expressed Foxp3. As expected, we first detect the expression of Foxp3 in PANC-1 cells by cell flow. We found that $11 \%$ of PANC-1 cells expressed Foxp3 (Fig. 1B).

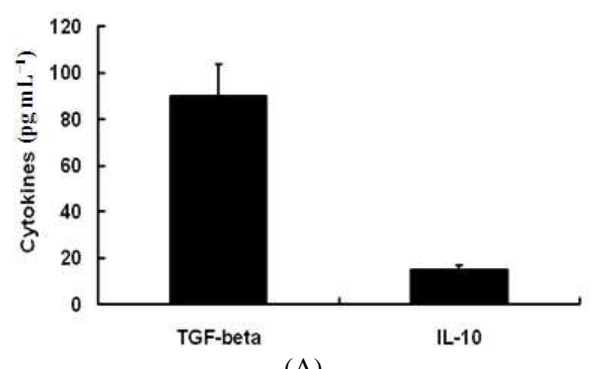

(A)

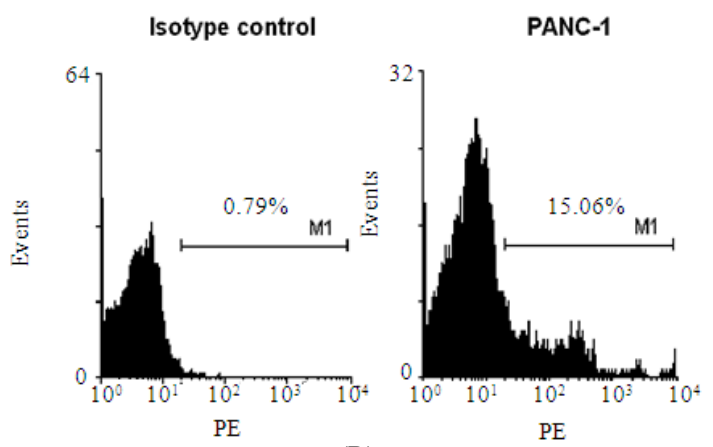

(B)

Fig. 1: Foxp3 expression in PANC-1 cells

Note: PANC-1 cells were cultured. After $48 \mathrm{~h}$, cells and supernatant were collected. (A) The production of IL-10 and TGF- $\beta$ was determined by ELISA. (B) Cells were stained for Foxp3 and analyzed by flow cytometry. The percentage of Foxp3+ cells was showed. The results are representative of four separate experiments
Foxp3 suppressed IL-10 and TGF- $\beta$ in PANC-1 cells: Whether does Foxp3 as a suppressive transcription factor suppress TGF- $\beta$ expression? Firstly, we set up SiRNA assays to knock down Foxp3 expression. By flow cytometry, we found that the percentage of Foxp3-expressing PANC-1 cells decreased from $2-11 \%$ (Fig. 2A).

Next, we determined the effect of Foxp3 downregulation on IL-10 and TGF- $\beta$ synthesized by PANC1. PANC-1 cells expressed much higher level of TGF- $\beta$ and IL-10 when Foxp3 was knocked down by SiRNA assays (Fig. 2B). These results suggest that Foxp3 expression suppressed TGF- $\beta$ and IL-10 expression in PANC-1 cells.
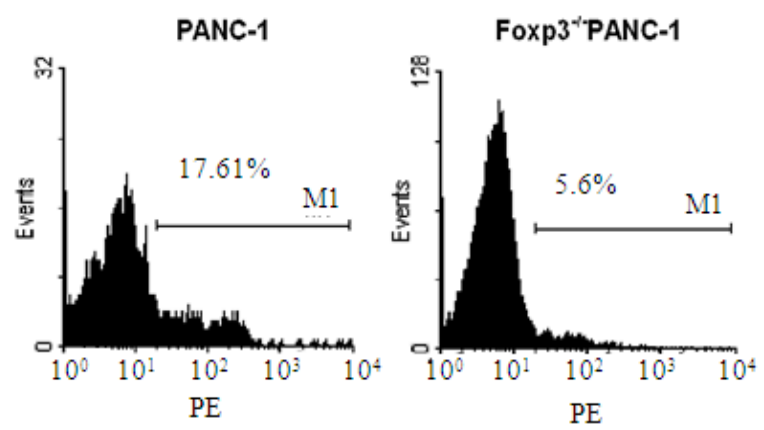

(A)

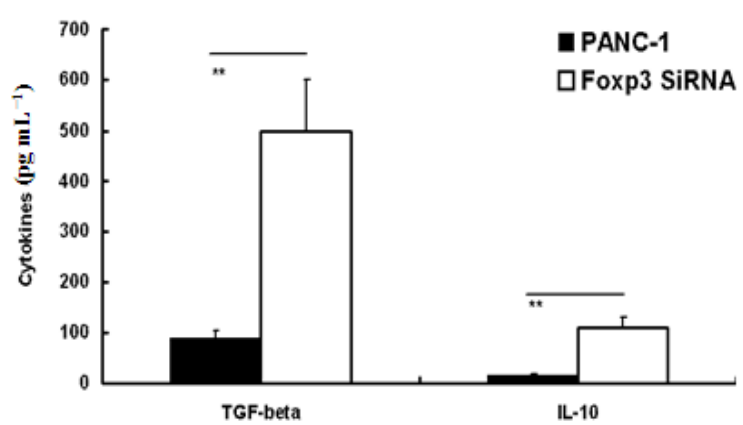

(B)

Fig. 2: Foxp3 suppressed TGF- $\beta$ and IL-10 expression in PANC-1 cells

Note: Human Foxp3 siRNAs were used to transduce PANC-1 cells (called as Foxp ${ }^{--}$PANC-1 cells). At $48 \mathrm{~h}$ after transduction, cells and supernatant were collected. (A) Cells were stained for Foxp3 and analyzed by flow cytometry. The percentage of Foxp $3^{+}$cells was showed. (B) The production of IL-10 and TGF- $\beta$ was determined by ELISA. The results are representative of three separate experiments $(* *: p \cdot 0.01)$ 
PANC-1 cells induced tolerance and response model: Thus, we propose that in PANC-1 cells, Foxp3 may be an important factor inducing tumor immunity by suppressing TGF- $\beta$ and IL-10 expression. To prove the proposal, we first develop PANC-1 cells induced tolerance and immunity model. The autoimmune NonObese Diabetic (NOD) mouse, characterized by T-cell mediated destruction of the insulin-producing $\beta$-cells in the pancreatic islets, were used ${ }^{[17]}$. About $1 \times 10^{7}$ PANC1 cells were injected into 7-weeks-old female NOD mice. As shown in Fig. 3A, the suppression of diabetes in PANC-1-treated group persisted for 18 weeks, compared to recipients of the control $(\mathrm{p}<0.05)$. After 24 weeks of age, PANC-1-treated NOD mice showed higher diabetes incidence than that in control groups $(\mathrm{p}<0.05)$. About $1 \times 10^{7}$ PANC- 1 cells were injected into 12 weeks-old female NOD mice. As shown in Fig. 3B, diabetes incidence was significantly higher in PANC-1treated NOD mice than that in control groups $(\mathrm{p}<0.01)$.

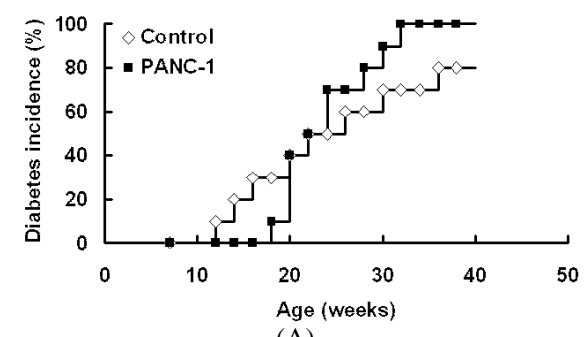

(A)

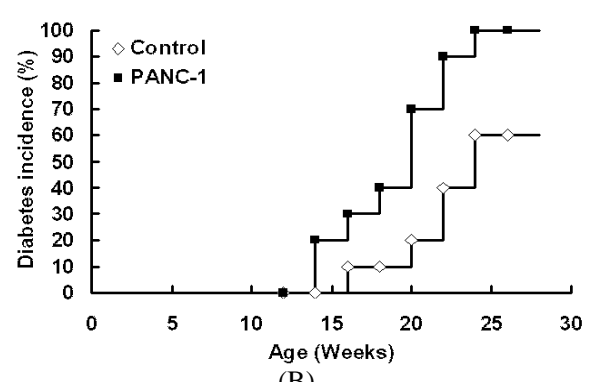

(B)

Fig. 3: PANC-1 cells were injected into autoimmune diabetic NOD mice

Note: (A) 7 week-old or (B) 12-week-old female NOD mice were injected with $1 \times 10^{7}$ PANC-1 cells per mouse ( $\diamond$ PANC-1, 10 mice per group) or Saline ( Control, 10 mice per group). Mice were screened for glucose levels every 1-2 weeks after gene therapy and considered diabetic when glucose levels $13.8 \mathrm{mMol} \mathrm{L}^{-1}$ were detected at two consecutive measurements. Results of diabetes incidence (number of diabetic out of total mice) represent a typical experiment
Above results makes us propose that PANC-1 cells induce immune tolerance of splenocytes from 7-weekold female NOD mice and response of splenocytes from 12-week-old female NOD mice to autoantigen Glutamic Acid Decarboxylase 65 (GAD65 ${ }^{[18]}$. We used splenocytes from 7 or 12-week-old female NOD mice and PANC-1 cells to co-culture in vitro. As expected, PANC-1 induced immune tolerance of splenocytes from 7 week-old female NOD mice and response of splenocytes from 12 week-old female NOD mice to $\mathrm{GAD}_{500-585}$ (Fig. 4A). In the parallel with cell proliferation, PANC-1 cells induced anti-inflammatory cytokines IL-10, TGF- $\beta$ and suppressed inflammatory
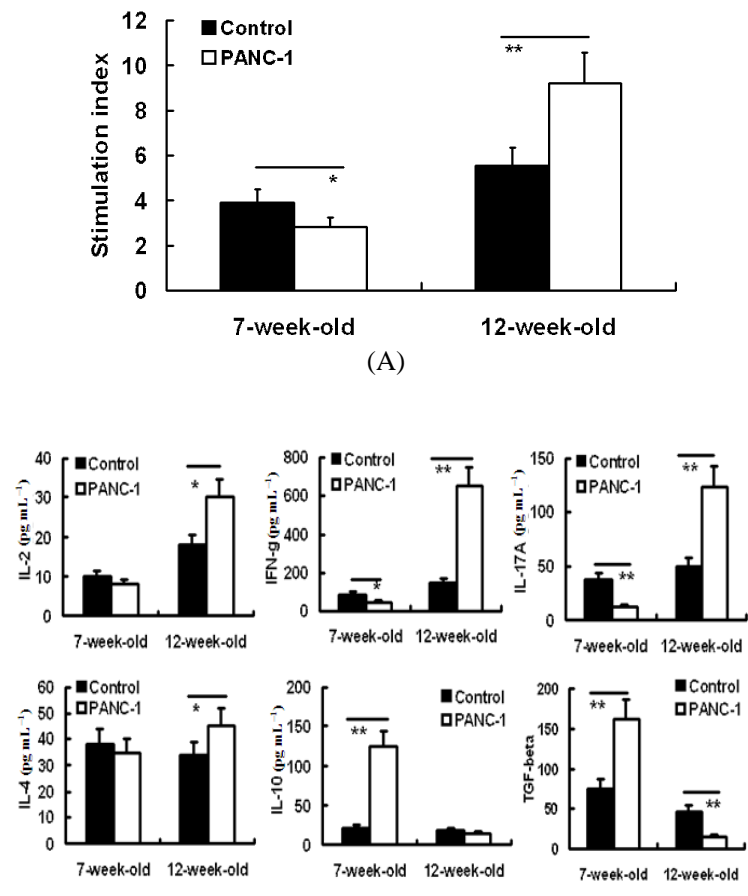

(B)

Fig. 4: PANC-1-induced tolerance and response model Note: Splenocytes were collected from 7 or 12 weeksold female NOD mice and stimulated for $48 \mathrm{~h}$ with $30 \mu \mathrm{g} \mathrm{mL}^{-1} \mathrm{GAD}_{500-585}$ without or in the presence of PANC-1 (PANC-1). (A) Cell proliferation was measured by incorporation of $[3 \mathrm{H}]$ thymidine during the final $16 \mathrm{~h}$ of culture. Data are presented as stimulation index (the ratio of c.p.m. with Ag to c.p.m. with medium alone) with the background of 1000- 3000 c.p.m. (B) The production of IL-2, IFN- $\gamma$, IL-4, IL-17A, IL10 and TGF- $\beta$ were determined by sandwich ELISA in the co-culture supernatants. The results are representative of three separate experiments, $(* *: \mathrm{p}<0.01$ and $*: \mathrm{p}<0.05)$ 


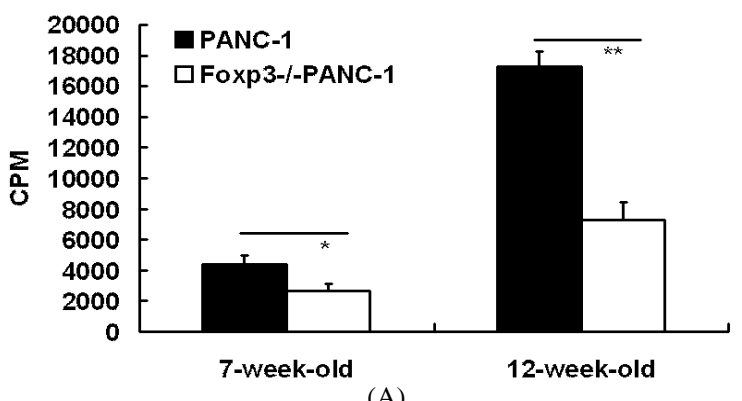

(A)
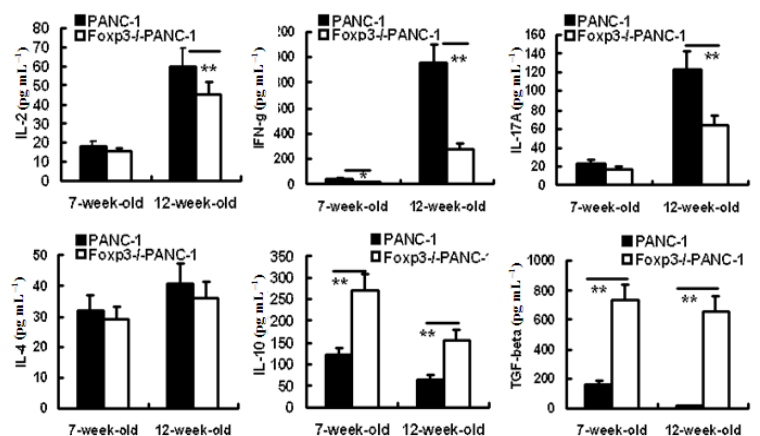

(B)

Fig. 5: Foxp3 determined PANC-1 cells-inducing immunity

Note: PANC-1 cells were transduced with human Foxp3 SiRNA, after $48 \mathrm{~h}$, Foxp3 SiRNAtransduced PANC-1 cells (Foxp3 ${ }^{-/}$PANC-1) were used to co-culture for $48 \mathrm{~h}$ with splenocytes from 7 or 12-weeks-old female NOD mice in the presence of $30 \mu \mathrm{g} \mathrm{mL}$ $\mathrm{GAD}_{500-585}$. (A) Cell proliferation was measured by incorporation of $[3 \mathrm{H}]$ thymidine during the final $16 \mathrm{~h}$ of culture. Data are presented as c.p.m. (B) The production of IL-2, IFN- $\gamma$, IL-4, IL-17A, IL-10 and TGF- $\beta$ were determined by sandwich ELISA in the co-culture supernatants. The results are representative of three separate experiments, $(* *: p<0.01$ and $*$ : $p<0.05)$

cytokine IL-2, IFN- $\gamma$, and IL-17A in GAD $_{500-585^{-}}$ stimulated splenocytes from 7-week-old NOD, whereas PANC-1 cells induced inflammatory cytokine IL-2, IFN- $\gamma$, and IL-17A and suppressed anti-inflammatory cytokines IL-10, TGF- $\beta$ in GAD $_{500-585}$-stimulated splenocytes from 12 week-old NOD response (Fig. 4B). These results suggest that co-cultured PANC-1 and $\mathrm{GAD}_{500-585}$-activated splenocytes from 7 week-old female NOD mice could be as PAN-1-induced tolerance model and co-cultured PANC-1 and GAD $_{500-585}{ }^{-}$ activated splenocytes from 12 week-old female NOD mice could be as PAN-1-induced immune model.
Foxp3 were critical for PANC-1 cells immunity: To further show the role of Foxp 3 in PANC-1 cells, we use Foxp $3^{-/}$PANC-1 cells (Foxp3 expression was knocked down in PANC-1 cells by SiRNA assays) to co-culture with splenocytes from 7 or 12-week-old female NOD mice in the presence of $\mathrm{GAD}_{500-585}$. We found that Foxp $3^{-/}$PANC-1 cells induced tolerance in $\mathrm{GAD}_{500-585^{-}}$ stimulated splenocytes from 7 or 12-week-old female NOD mice (Fig. 5A). In addition, Foxp3 $3^{-/}$PANC-1 cells upregulated anti-inflammatory cytokines IL-10, TGF- $\beta$ and downregulated inflammatory cytokine IL-2, IFN$\gamma$ in $\mathrm{GAD}_{500-585}$-stimulated splenocytes from 7 or 12 week-old female NOD mice (Fig. 5B). These results suggest that Foxp3 is an important factor for PANC-1 cells-induced immune response.

\section{DISCUSSION}

Foxp3 expression has been thought to be restricted to the T-cell lineage. Until recently, several reports were published about Foxp3 expression in human cancer cells ${ }^{[7-9]}$. In these studies, they showed Foxp3 expression in many tumor cell lines of different tissues origins by RT-PCR and Western blot assay. We also detected Foxp3 in human pancreatic cancer cell line PANC-1 by cell flow (Fig. 1B). Except for in tumor cells, Foxp3 is expressed in normal breast epithelium $^{[10]}$.

TGF- $\beta$ and IL-10 were factors most frequently released by pancreatic ductal carcinoma cell line ${ }^{[11]}$. We also found that PANC-1 expressed TGF- $\beta$ and IL-10 (Fig. 1A). TGF- $\beta$ can converse peripheral $\mathrm{CD} 4^{+} \mathrm{CD} 25^{-}$ naive $\mathrm{T}$ cells to $\mathrm{CD} 4^{+} \mathrm{CD} 25^{-}$regulatory $\mathrm{T}$ cells by inducing Foxp3 expression ${ }^{[16]}$. These results suggest that auto-secreted TGF- $\beta$ may induce Foxp 3 expression in PANC-1 cells. A report found that Foxp3 expression was induced by TGF- $\beta 2$, but not TGF- $\beta 1$ stimulation in pancreatic ductal adenocarcinoma cells ${ }^{[7]}$.

The role of Foxp3 in TGF- $\beta$ expression is still unclear. We have known that Foxp3 is a suppressive transcription factor ${ }^{[19]}$. Foxp3 suppress the transcription of several target genes including IL-2, IFN- $\gamma$, IL-4, and IL-10 $0^{[1,6]}$ in Treg, HER-2/ErbB2 oncogene expression in breast cancer ${ }^{[16]}$, IL-6 and IL-8 in pancreatic carcinoma cell line PANC-89 ${ }^{[7]}$. Can Foxp3 as suppressive transcription factor suppress TGF- $\beta$ expression? Foxp3expressing $\mathrm{CD} 4^{+} \mathrm{CD} 25^{+}$Treg cells-mediated tolerance is dependent on TGF- $\beta$-dependent ${ }^{[20]}$ suggesting that Foxp3 may promote TGF- $\beta$ expression. Foxp3 expression was knocked down in PANC-1 cells by SiRNA assays to study the role of Foxp3 in TGF- $\beta$ secreted by PANC-1. TGF- $\beta$ and IL-10 were upregulated in PANC-1 cells when Foxp3 was knocked 
down (Fig. 2B). These results suggest that Foxp3 expression suppressed TGF- $\beta$ and IL-10 expression in PANC-1 cells.

To further explore the role of Foxp3 in the immunity of PANC-1 cells, we develop the PANC-1 induced tolerance and immunity model. The NOD strain of mouse is an increasingly useful and important model of autoimmune type 1 diabetes ${ }^{[17]}$. The autoimmune disease characterized by progressive destruction of pancreatic beta cells we found that PANC-1 cells could control diabetes until 13 weeks after therapy when injected into 7-weeks-old female NOD mice (Fig $3 \mathrm{~A})$. In vitro experiment further proved that PANC-1 cells induced tolerance (Fig. 4A) and immunoregulatory cytokines IL-10 and TGF- $\beta$, and suppressed inflammatory cytokine IL-2, IFN- $\gamma$ and IL17 in splenocytes from 7 week-old female NOD mice (Fig. 4A and B). These results show that PANC-1 cells induced tolerance in splenocyte from 7 weeks-old female NOD mice. Tumor cells employed different strategies to thwart immune responses: tumor-induced impairment of antigen presentation, the activation of negative co-stimulatory signals, and the elaboration of immunosuppressive factors ${ }^{[12]}$. IL-10 and TGF- $\beta$ are immunoregulatory cytokines that are involved in induction and maintenance of peripheral tolerance by affecting autoreactive T-cell-mediated response ${ }^{[21-23]}$. The studies suggest that PANC-1 cells induce immune tolerance partly by auto-secreted TGF- $\beta$ and IL- 10 .

When PANC-1 cells were injected into 12 weeksold female NOD mice, diabetes incidence was significantly higher in PANC-1-treated NOD mice (Fig. 3B). In vitro experiment further proved that PANC-1 cells induced response (Fig. 4A) and inflammatory cytokine IL-2, IFN- $\gamma$ and IL-17, and suppressed immunoregulatory cytokines IL-10 and TGF- $\beta$ in splenocytes from 12 week-old female NOD mice (Fig. 4B). The fact that most mice demonstrate severe insulitis by 10 weeks of age explained that inflammatory response is very strong in 12 weeks-old female NOD mice. These results suggest that PANC-1 cells induced immune response in splenocytes from 12week-old female NOD mice. We did not know the reason for inflammatory response induced by PANC-1. The PANC-1 cells were injected into NOD mice and they maybe difficultly form tolerance microenvironment in autoimmune condition. Thus, they may induce response over tolerance in autoimmune condition.

PANC-1-induced immune response and tolerance model were very useful in studying the role of Foxp3 in immunity of PANC- 1 cells. In the different condition, the response of cells and the molecular expression and function may vary. There are PANC-1-induced immune response and tolerance model make us gain more information in the function of Foxp3. We found that Foxp $3^{-1}$ PANC-1 cells significantly induced tolerance in $\mathrm{GAD}_{500-585}$-stimulated splenocytes from 12 week-old and 7-week-old female NOD mice (Fig. 5A). In addition, Foxp $3^{-1}$ PANC-1 cells induced antiinflammatory cytokines IL-10, TGF- $\beta$ and suppressed inflammatory cytokine such as IL-2, IFN- $\gamma$ and IL-17A in $\mathrm{GAD}_{500-585}$-stimulated splenocytes from 12-week-old and 7 week-old female NOD mice (Fig. 5B). These results suggest that Foxp3 control the capacity of PANC-1 cells in inducing immune response. In addition, the extent to reducing response in PANC-1induced response model was obviously bigger than that in PANC-1-induced tolerance model. This may explain for the necessary of building PANC-1-induced response model.

\section{CONCLUSSION}

In conclusion, human pancreatic cancer cell line PANC-1 exhibits immunological response characteristics by expressing Foxp3. The effect may act partly by suppressing TGF- $\beta$ expression. Thus, we proved our proposal that Foxp3 may be an important factor inducing tumor immunity by suppressing TGF- $\beta$ expression in PANC-1 cells. It is under study that immunogenic response of PANC-1 cells is enhanced by Foxp3 over-expression.

\section{ACKNOWLEDGEMENT}

We gratefully acknowledge Dr. Jiannan Feng for useful advice. This work was supported by National Nature and Science Funds (30571732) and National “973" Fund Grant 2007CB512406 and 2009CB522408. The authors have no conflicts of interest.

\section{REFERENCES}

1. Hori, S., T. Nomura and S. Sakaguchi, 2003. Control of regulatory $\mathrm{T}$ cell development by the transcription factor Foxp 3. Sci., 299: 1057-1061. http://www.ncbi.nlm.nih.gov/pubmed/12522256

2. Ramsdell, F. and S.F. Ziegler, 2003. Transcription factors in autoimmunity. Curr. Opin. Immunol., 15: 718-724.

http://www.ncbi.nlm.nih.gov/pubmed/14630208

3. Fontenot, J.D., M.A. Gavin and A.Y. Rudensky, 2003. Foxp3 programs the development and function of $\mathrm{CD} 4+\mathrm{CD} 25+$ regulatory $\mathrm{T}$ cells. Nat. Immunol., $\quad$ 3: 330-336. http://www.ncbi.nlm.nih.gov/pubmed/12612578 
4. Yagi, H., T. Nomura, K. Nakamura, S. Yamazaki and T. Kitawaki et al., 2004. Crucial role of FOXP3 in the development and function of human CD25+CD4+ regulatory $\mathrm{T}$ cells. Int. Immunol., 16: 1643-1656. PMID: 15466453

5. Allan S.E., L. Passerini, R. Bacchetta, N. Crellin and M. Dai et al., 2005. The role of 2 FOXP3 isoforms in the generation of human CD4+ Tregs. J. Clin. Invest., 115: 3276-3284. PMID: 16211090

6. Wu, Y., M. Borde, V. Heissmeyer, M. Feuerer and A.D. Lapan et al., 2006. FOXP3 controls regulatory $\mathrm{T}$ cell function through cooperation with NFAT. Cell, 126: 375-387. PMID: 16873067

7. Hinz, S., L. Pagerols-Raluy, H.H. Oberg, O. Ammerpohl and S. Grüssel et al., 2007. Foxp3 expression in pancreatic carcinoma cells as a novel mechanism of immune evasion in cancer. Cancer Res., 67: 8344-8350.

http://cancerres.aacrjournals.org/cgi/content/abstrac $\mathrm{t} / 67 / 17 / 8344$

8. Ebert, L.M, B.S. Tan, J. Browning, S. Svobodova and S.E. Russell et al., 2008. The regulatory T cellassociated transcription factor foxp 3 is expressed by tumor cells. Cancer Res., 68: 3001-3009. http://www.ncbi.nlm.nih.gov/pubmed/18413770

9. Karanikas, V., M. Speletas, M. Zamanakou, F. Kalala and G. Loules et al., 2008. Foxp3 expression in human cancer cells. J. Trans. Med., 6: 19. PMID: PMC2386447/

10. Zuo, T., L. Wang, C. Morrison, X. Chang and H. Zhang et al., 2007. Foxp3 is an X-linked breast cancer suppressor gene and an important repressor of the HER-2/ErbB2 oncogene. Cell, 129: 1275-1286. http://www.ncbi.nlm.nih.gov/pubmed/17570480

11. Monti, P., F. Marchesi, M. Reni, A. Mercalli, V. Sordi et al., 2004. A comprehensive in vitro characterization of pancreatic ductal carcinoma cell line biological behavior and its correlation with the structural and genetic profile. Virchows Archl., 445: 236-247.

http://cat.inist.fr/?aModele $=$ afficheN\&cpsidt $=16092244$

12. Rabinovich, G.A, D. Gabrilovich and E.M. Sotomayor, 2007. Immunosuppressive strategies that are mediated by tumor cells. Annu. Rev. Immunol., 25: 267-296. PMID: 17134371

13. Waldmann, T.A., 2006. Effective cancer therapy through immunomodulation. Annu. Rev. Med., 57: 65-81. PMID: 16409137

14. Li, M.O., Y.Y. Wan, S. Sanjabi, A.K. Robertson and R.A. Flavell, 2006. Transforming growth factor- $\beta$ regulation of immune responses. Annu. Rev. Immunol., 24: 99-146. http://www.ncbi.nlm.nih.gov/pubmed/16551245
15. Huber, S., C. Schramm, H.A. Lehr, A. Mann and S. Schmitt et al., 2004. Cutting edge: TGF-beta signaling is required for the in vivo expansion and immunosuppressive capacity of regulatory CD4+CD25+ T cells. J. Immunol., 173: 6526-6531. http://www.ncbi.nlm.nih.gov/pubmed/15557141

16. Chen, W., W. Jin, N. Hardegen, K.J. Lei and L. Li et al., 2003. Conversion of peripheral CD4+CD25- naive $\mathrm{T}$ cells to CD4+CD25+ regulatory $\mathrm{T}$ cells by TGFbeta induction of transcription factor Foxp3. J. Exp. Med., 198: 1875-1886. PMID: 14676299

17. Anderson, M.S. and J.A. Bluestone, 2005. The NOD mouse: A model of immune dysregulation. Annu. Rev. Immunol., 23: 447-485.

18. Lieberman, S.M. and T.P. DiLorenzo, 2003. A comprehensive guide to antibody and T-cell responses in type 1 diabetes. Tissue Antigens, 62: 359-377. PMID: 15771578

19. Schubert, L.A., E. Jeffery, Y. Zhang, F. Ramsdell and S.F. Ziegler, 2001. Scurfin (FOXP3) acts as a repressor of transcription and regulates $\mathrm{T}$ cell activation. J. Biol. Chem., 276: 37672-37679. http://www.ncbi.nlm.nih.gov/pubmed/11483607

20. Mucida, D., N. Kutchukhidze, A. Erazo, M. Russo, J.J. Lafaille and M.A. Curotto de Lafaille, 2005. Oral tolertance in the absence of naturally occurring Tregs. J. Clin. Invest., 115: 1923-1933. http://cat.inist.fr/?aModele $=$ afficheN\&cpsidt $=1701$ 7445

21. Hill, N. and N. Sarvetnick, 2002. Cytokines: Promoters and dampeners of autoimmunity. Curr. Opin. Immunol., 14: 791-797. PMID: 12413531

22. Roncarolo, M.G., R. Bacchetta, C. Bordignon, S. Narula and M.K. Levings, 2001. Type 1 T regulatory cells. Immunol. Rev., 182: 68-79. PMID: 11722624

23. Weiner, H.L., 2001. Induction and mechanism of action of transforming growth factor-b secreting Th3 regulatory cells. Immunol. Rev., 182: 207-214. http://www.ncbi.nlm.nih.gov/pubmed/11722636

24. Song, L., J. Wang, R. Wang, M. Yu and Y. Sun et al., 2004. Retroviral delivery of GAD-IgG fusion construct induces tolerance and modulates diabetes: A role for $\mathrm{CD}^{+}$regulatory $\mathrm{T}$ cells and TGF-beta? Gene Ther., 11: 1487-1496. http://cat.inist.fr/?aModele=afficheN\&cpsidt $=16147527$

25. Han, G., Y. Li, J. Wang, R. Wang and G. Chen et al., 2005. Active tolerance induction and prevention of autoimmune diabetes by immunogene therapy using recombinant adenoassociated virus expressing glutamic acid decarboxylase 65 peptide GAD (500-585). J. Immunol., 174: 4516-4524. http://www.jimmunol.org/cgi/content/abstract/174/ $8 / 4516$ 